\title{
Transmission Delay Analysis with Finite Coding Length in Wireless Cooperative Networks
}

\author{
Zhengguo Sheng*, Zhiguo Ding $\dagger$, and Kin K Leung* \\ *Department of Electrical and Electronic Engineering, Imperial College, UK \\ $\dagger$ Department of Communication Systems, Lancaster University, UK \\ Email: zhengguo.sheng06@imperial.ac.uk
}

\begin{abstract}
In this paper, we consider the problem of transmission delay in terms of finite coding length derived from the random coding bound for different cooperative protocols. Specifically, we first study the impact of cooperative transmission on the routing decision for wireless ad-hoc networks, where a routing optimization problem is formulated to minimize the endto-end delay that ensures a satisfactory error performance. The closed-expression of the optimal solution is developed through the optimization problem and later used as quantitative criterion of routing decision. Furthermore, by considering the interference impact on system performance along a multi-hop routing, we then investigate performance gain on transmission delay for wireless cooperative networks by using a simple multi-user detection scheme, called overlapped transmission, in which multiple transmissions are allowed only when the information in the interfering signal is known at the receiver. As a result, both analytical and numerical results demonstrate the significant improvement on the system performance by using cooperative transmission with overlapping as well as the trade-off between the end-to-end delay and network throughput.
\end{abstract}

\section{INTRODUCTION}

Cooperative transmission (CT) has gained much attention as an effective technique to combat multi-path fading and enhance receiver reliability of wireless communication systems. The key feature of cooperative transmission is to encourage single-antenna devices to cooperatively share their antennas such that a virtual antenna array can be constructed and, hence significantly boosting reception reliability. Due to the broadcasting nature of wireless communications, cooperative transmission has been recognized as an effective technique to combat multi-path fading, save energy and enhance receiver reliability in wireless communication systems [1].

In this paper, we consider a different question in the context of cooperative communication to analyze the performance gain on transmission delay when using cooperative transmission. To our best knowledge, the effect of cooperative transmission on the delay performance is first studied in this paper. According to the unique features of different cooperative protocols (e.g., amplify-forward, decode-forward and multi-hop), we use the

Research was sponsored by US Army Research laboratory and the UK Ministry of Defence and was accomplished under Agreement Number W911NF06-3-0001. The views and conclusions contained in this document are those of the authors and should not be interpreted as representing the official policies, either expressed or implied, of the US Army Research Laboratory, the U.S. Government, the UK Ministry of Defense, or the UK Government. The US and UK Governments are authorized to reproduce and distribute reprints for Government purposes notwithstanding any copyright notation hereon. error exponent model [2] to formulate the random coding bound for each protocol. It is shown that cooperative transmission can improve the quality of a wireless link and thus significantly reduce the transmission delay without adversely affecting the reliability.

The problem of routing optimization is then investigated in order to study the effect of improved link cost on the routing decision. The objective function under study is to minimize the end-to-end delay with a constrained end-to-end reliability. The optimal solution indicates the minimum end-to-end delay of a route in order to satisfy the required error performance, which is then used as a criterion to compare and select the best protocol under different network scenarios. Meanwhile, the developed criterion also implies that it is preferable to choose a multiple hops route which has as many links using cooperative transmission as possible.

Furthermore, in order to investigate the interference impact on network performance, we propose here to further exploit delay performance in cooperative networks by implementing the Multi-User Detection (MUD) scheme [3] under a more realistic network scenario which allows multi-node transmissions along the same route using space time reuse scheme. Analytical result shows that the cooperative scheduling employing overlapped transmission achieves much better delay performance than that without employing overlapped transmission. In order to obtain more insights on the relation between the delay and other system performance parameter (e.g., throughput), we consider here to minimize the end-toend delay and maximize the average throughput, respectively. Through optimizing the block length as well as the channel reuse factor for sending a fixed size data from the source to the destination, the trade-off between the delay and network throughput is also revealed by the developed analytical and simulation results in the end.

\section{System Model}

In this paper, we consider a wireless ad-hoc network where nodes are assumed to be uniformly distributed. Each node is equipped with one omnidirectional antenna element. The Time Division Multiple Access (TDMA) scheme is used to enable various nodes to share the same frequency band. To evaluate average performance in the network, we employ a propagation model to only consider distance dependent pathloss and additive Gaussian noise [4]. The wireless link between 
the nodes $i$ and $j$ is modelled as $\mathrm{a}_{i j}=d_{i j}^{-\alpha / 2}$, where $d_{i j}$ is the distance between the nodes $i$ and $j$, represents the large-scale behavior of the channel gain and $\alpha$ is the path-loss exponent.

It is assumed that each node uses a random Gaussian code ${ }^{1}$ to encode a block of $L$ nats $^{2}$ information into a time signal of infinite duration (henceforth codeword) and transmit it. However, the transmitter will transmit a finite time only until the receiver successfully decodes the message, thus only a finite length of codeword will be transmitted. From [2], [5], [6], since the Gaussian waveform channel can be modelled as a sequence of complex Gaussian scalar channels, if we use the output of the first $N$ channels to decode the transmitted message (i.e., decoding at time $N / W$, where $N$ is number of samples used for decoding), the one hop coding bound on block error probability $P_{e}$ is

$$
P_{e} \leq \exp \left(\rho L-N\left(E_{0}(\rho, \mathrm{SINR})\right),\right.
$$

with normalized bandwidth $W=1$ for any $\rho \in[0,1]$. SINR is the Signal-to-Interference-Plus-Noise ratio at the receiver and it is also assumed to be the same during the one block transmission, and $E_{0}(\rho, \mathrm{SINR})$ is the error exponent determined by $\rho$ and SINR. For a complex Gaussian channel with unit bandwidth, a simple expression for the error exponent is derived from [2]

$$
E_{0}(\rho, \operatorname{SINR})=\rho \ln \left(1+\frac{\mathrm{SINR}}{1+\rho}\right),
$$

Given a target block error probability $P_{e}$ that the receiver can successfully decode the message, the minimum $N$ per hop is bounded by

$$
N_{p h} \geq \frac{\rho L-\ln P_{e}}{\rho \ln \left(1+\frac{\operatorname{SINR}}{1+\rho}\right)} .
$$

The lower bound is the minimum coding length for sending $L$ nats information over that hop when a target reliability constraint is guaranteed. Given the decoding time is $N / W$, we will use this lower bound as the minimum delay to evaluate the network performance in the rest of paper. It is worth noting that we only consider the transmission delay for the sake of simplicity. This is a sensible assumption, we may imagine the given flow has higher priority over other flows in the network.

\section{Link DELAy Using CoOperative Transmission}

We consider a cooperative link (CL), where a source node communicates with a destination node with the help of one relay. In the following, we characterize delay performance of three low-complexity cooperative protocols that can be utilized in the network, including amplify-forward, decode-forward and classic multi-hop [7]. Each of them employs a different type of processing technique by the relay node, as well as different type of combining at the destination.

\footnotetext{
${ }^{1}$ The Gaussian code encoder separates the incoming binary data stream into equal length of $L$ binary digits each. There are total $M=2^{L}$ different binary sequences of length $L$ and the encoder provides a codeword for each. Each codeword is a sequence of a fixed number, $N$, of channel input letters. The codewords are samples of bandlimited white Gaussian noise.

${ }^{2}$ In order to simplify notation and analysis, we use information unit nat in this paper; 1 nat $=\log _{2}$ ebit
}

\section{A. Amplify-Forward}

For amplify-forward (AF) transmission, the relay node amplifies whatever its received subject to its power constraint and retransmits the signals to the destination. As explained in detail in [1], the maximum average mutual information between the source and the destination is given by

$$
I_{A F}=\frac{1}{2} \log \left(1+\operatorname{SINR}_{s, d}+f\left(\operatorname{SINR}_{s, r}, \operatorname{SINR}_{r, d}\right)\right),
$$

where SINR is defined as received power to noise plus interference ratio and $f(x, y)=\frac{x y}{x+y+1}$.

According to (3), the delay performance of amplify-forward is derived as

$$
D_{A F} \geq \frac{2\left(\rho L-\ln P_{e}\right)}{\rho \ln \left(1+\frac{\operatorname{SINR}_{s, d}+f\left(\operatorname{SINR}_{s, r}, \operatorname{SINR}_{r, d}\right)}{1+\rho}\right)} .
$$

Since the relay transmission requires two equal time slots, the extra factor of 2 is added in the delay.

\section{B. Decode-Forward}

As compared with amplify-forward, decode-forward (DF) allows the relay to decode the signals from the source, reencode and retransmit the signals to the destination. Specifically, here we consider the Selection Decode-Forward [1] transmission. Let $d_{s, d}, d_{s, r}$ and $d_{r, d}$ be the respective distances among the source, relay and destination. During the first time slot, the destination receives $y_{d}=\frac{1}{d_{s, d}^{\alpha / 2}} x_{s}+n_{d}$ from the source node, where $x_{s}$ is the transmitted signal from the source and $n_{d}$ is white noise. During the second time slot, the destination node receives

$$
y_{d}= \begin{cases}\frac{1}{d_{s, d}^{\alpha / 2}} x_{s}+n_{d}, & \text { if } R>I_{s, r} \\ \frac{1}{d_{r, d}^{\alpha / 2}} x_{r}+n_{d}, & \text { if } R \leq I_{s, r}\end{cases}
$$

where $R=L /\left(2 N_{p h} \ln 2\right) \mathrm{bit} / \mathrm{s} / \mathrm{unit}$ hertz $^{3}$ and $I_{s, r}=$ $\frac{1}{2} \log \left(1+\operatorname{SINR}_{s, r}\right)$ can be derived from direct transmission. As can be seen from the first condition of (6), when the link between the source and the relay is so poor that the relay is not able to decode, there is no performance gain can be achieved and the source is repeating its transmission during this slot. The second condition corresponds to the case when the relay can decode and repeat the source transmission. As a result, the destination receives two independent copies of the same packets transmitted through different wireless channels, thus obtaining the second-order diversity gain through CL.

Consider that a relay node is randomly selected, hence the delay performance of decode-forward transmission is shown as

$$
D_{D F} \geq \begin{cases}2 \frac{\rho L-\ln P_{e}}{\rho \ln \left(1+\frac{2 \operatorname{SINR}_{s, d}}{1+\rho}\right)}, & \text { if } R>I_{s, r} \\ 2 \frac{\rho L-\ln P_{e}}{\rho \ln \left(1+\frac{\operatorname{SINR}_{s, d}+\operatorname{SINR} r, d}{1+\rho}\right)}, & \text { if } R \leq I_{s, r}\end{cases}
$$

\footnotetext{
${ }^{3}$ Since we use the information nat as the unit in this paper, the original data rate $R=\frac{L}{N W \tau_{t}}$ in nat/s/unit hertz should be converted to that in bit/s/unit hertz. The sampling time $\tau_{t}$ at the decoder equals Nyquist rate $W$ of 1 unit time per symbol.
} 


\section{Classic Multi-Hop}

Classic multi-hop $(\mathrm{MH})$ has the source transmitting its signals to the relay in one time slot, and then the relay forwarding the signals to the destination in a second time slot. In order to derive its delay performance, we formulate an optimization problem to minimize the link delay with a constrained block error probability $P_{e}$ as is shown

$$
\begin{array}{cl}
\min & D_{s, r}+D_{r, d} \\
\text { s.t. } & 1-\left(1-P_{s, r}\right)\left(1-P_{r, d}\right) \leq P_{e}
\end{array}
$$

Then the delay performance of classic multi-hop can be derived as

$$
D_{M H} \geq \frac{\rho L-\ln \left(\frac{P_{e} K_{s, r}}{K_{s, r}+K_{r, d}}\right)}{\rho \ln \left(1+\frac{\operatorname{SINR}_{s, r}}{1+\rho}\right)}+\frac{\rho L-\ln \left(\frac{P_{e} K_{r, d}}{K_{s, r}+K_{r, d}}\right)}{\rho \ln \left(1+\frac{\operatorname{SINR}_{r, d}}{1+\rho}\right)} .
$$

where $K_{i, j}=\frac{1}{\rho \ln \left(1+\frac{\operatorname{SINR}_{i, j}}{1+\rho}\right)}$. The development of (9) is similar to that in the Section IV and can refer to Appendix A.

\section{Route Optimization}

Based on the system model we defined in the previous section, now we return to the problem of delay analysis by first characterizing the minimum end-to-end delay for a multi-hop route. A meaningful optimization problem is to minimize the end-to-end delay in cooperative networks that ensures the endto-end error performance satisfied the target level (constraint).

Consider that a route has been constructed between the source and destination. Without losing generality, the nodes sitting on the route are denoted as $S \rightarrow 1 \ldots \rightarrow n \rightarrow D$. Different to traditional routes, cooperative transmission is used to improve the link quality. However, it is possible that a good helping relay is not available for some pairs of the $n+1$ links of the route. In that case, direct transmission (DT) is used instead of relying on cooperative transmission (CT). Hence the $n+1$ links involved in the route between the source and destination nodes can be categorized into two sets. The first set, defined as $\mathcal{S}_{1}$, includes the links using only direct transmission and the other one, defined as $\mathcal{S}_{2}$, includes all links using cooperative transmission. Note that $\left|\mathcal{S}_{1}\right|+\left|\mathcal{S}_{2}\right|=n+1$ since there are only $n+1$ links on the route.

The problem to minimize the end-to-end delay in cooperative networks using amplify-forward with the constraint on the end-to-end reliability can be formulated as

$$
\begin{array}{cl}
\min _{P_{i j}^{D T}, P_{i j}^{C T}} & \sum_{i j \in \mathcal{S}_{1}} D_{i j}^{D T}+\sum_{i j \in \mathcal{S}_{2}} D_{i j}^{C T} \\
\text { s.t. } & 1-\prod_{i j \in \mathcal{S}_{1}}\left(1-P_{i j}^{D T}\right) \prod_{i j \in \mathcal{S}_{2}}\left(1-P_{i j}^{C T}\right) \leq P
\end{array}
$$

For small block error probabilities $P_{i j}^{D T} \ll 1$ and $P_{i j}^{C T} \ll$ 1 , we can have the following approximation

$$
1-\prod_{i j \in \mathcal{S}_{1}}\left(1-P_{i j}^{D T}\right) \prod_{i j \in \mathcal{S}_{2}}\left(1-P_{i j}^{C T}\right) \approx \sum_{i j \in S_{1}} P_{i j}^{D T}+\sum_{i j \in S_{2}} P_{i j}^{C T}
$$

So the optimization problem can be simplified as

$$
\begin{array}{cl}
\min _{P_{i j}^{D T}, P_{i j}^{C T}} & \sum_{i j \in \mathcal{S}_{1}} D_{i j}^{D T}+\sum_{i j \in \mathcal{S}_{2}} D_{i j}^{C T} \\
\text { s.t. } & \sum_{i j \in S_{1}} P_{i j}^{D T}+\sum_{i j \in S_{2}} P_{i j}^{C T} \leq P
\end{array}
$$

By introducing an auxiliary variable $z,(12)$ can be written as

$$
\begin{array}{cl}
\min _{P_{i j}^{D T}, P_{i j}^{C T}, z} & \sum_{i j \in \mathcal{S}_{1}} D_{i j}^{D T}+\sum_{i j \in \mathcal{S}_{2}} D_{i j}^{C T} \\
\text { s.t. } & \sum_{i j \in \mathcal{S}_{1}} P_{i j}^{D T} \leq z, \sum_{i j \in \mathcal{S}_{2}} P_{i j}^{C T} \leq P-z \\
& 0 \leq z \leq P
\end{array}
$$

Hence the optimization problem can be solved in two stages. First we treat $z$ as a constant and solve the following two subproblems separately.

$$
\begin{array}{clll}
\min _{P_{i j}^{D T}} & \sum_{i j \in \mathcal{S}_{1}} D_{i j}^{D T} & \min _{P_{i j}^{C T}} & \sum_{i j \in \mathcal{S}_{2}} D_{i j}^{C T} \\
\text { s.t. } & \sum_{i j \in \mathcal{S}_{1}} P_{i j}^{D T} \leq z, & \text { s.t. } & \sum_{i j \in \mathcal{S}_{2}} P_{i j}^{C T} \leq P-z
\end{array}
$$

which yields the two solutions

$$
\begin{aligned}
\sum_{i j \in \mathcal{S}_{1}} D_{i j}^{D T} & =\sum_{i j \in \mathcal{S}_{1}} K_{i j}\left(\rho L-\ln \left(\frac{z K_{i j}}{\sum_{i j \in \mathcal{S}_{1}} K_{i j}}\right)\right) \\
\sum_{i j \in \mathcal{S}_{2}} D_{i j}^{C T} & =\sum_{i j \in \mathcal{S}_{2}} C_{i j}\left(\rho L-\ln \left(\frac{C_{i j}(P-z)}{\sum_{i j \in \mathcal{S}_{2}} C_{i j}}\right)\right)
\end{aligned}
$$

where $K_{i j}=\frac{1}{\rho \ln \left(1+\frac{\operatorname{SINR}_{i j}}{1+\rho}\right)}$ and $C_{i j}=$ $\frac{2}{\rho \ln \left(1+\frac{\operatorname{SINR}_{i, j}+f\left(\operatorname{SINR}_{i, r}, \operatorname{SINR}_{r, j}\right)}{1+\rho}\right)}$ for AF. The development of (15) and (16) is provided in Appendix A. Note that both $\sum_{i j \in \mathcal{S}_{1}} D_{i j}^{D T}$ and $\sum_{i j \in \mathcal{S}_{2}} D_{i j}^{C T}$ now become functions of the auxiliary variable $z$.

The second step is to solve the following optimization problem

$$
\begin{array}{cc}
\min _{z} & f_{z}(z)=\sum_{i j \in \mathcal{S}_{1}} K_{i j}\left(\rho L-\ln \left(\frac{z K_{i j}}{\sum_{i j \in \mathcal{S}_{1}} K_{i j}}\right)\right) \\
& +\sum_{i j \in \mathcal{S}_{2}} C_{i j}\left(\rho L-\ln \left(\frac{C_{i j}(P-z)}{\sum_{i j \in \mathcal{S}_{2}} C_{i j}}\right)\right) \\
\text { s.t. } & 0 \leq z \leq P
\end{array}
$$

Note that $f_{z}(z)$ is a convex function for $0 \leq z \leq P$ since $\frac{d^{2} f_{z}(z)}{d^{2} z}>0$. Hence there is only one minimum value for $0 \leq$ $z \leq P$ when $\frac{d f_{z}(z)}{d z}=0$. We can derive the following

$$
\frac{d f_{z}(z)}{d z}=\sum_{i j \in \mathcal{S}_{1}} \frac{K_{i j}}{z}-\sum_{i j \in \mathcal{S}_{2}} \frac{C_{i j}}{P-z}
$$

Then the optimal error probability distribution

$$
z^{*}=\frac{P \sum_{i j \in \mathcal{S}_{1}} K_{i j}}{\sum_{i j \in \mathcal{S}_{1}} K_{i j}+\sum_{i j \in \mathcal{S}_{2}} C_{i j}} .
$$




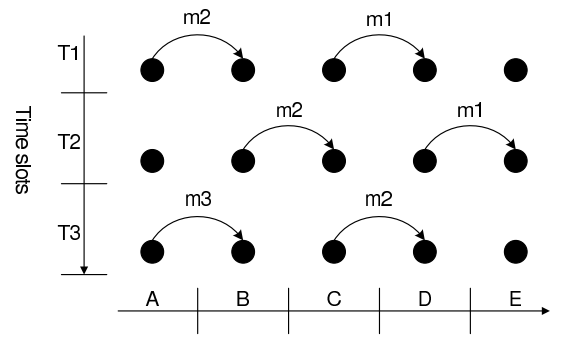

Fig. 1. Multi-hop scheduling $(K=2)$ with overlapped transmission in a five-node linear network

Finally putting $z^{*}$ into (17), we get the minimum end-to-end delay. Also from Appendix A, the optimal error probability distribution for each link can be shown as

$$
P_{i j}^{D T}=\frac{z^{*} K_{i j}}{\sum_{i j \in \mathcal{S}_{1}} K_{i j}}, \quad P_{i j}^{C T}=\frac{C_{i j}\left(P-z^{*}\right)}{\sum_{i j \in \mathcal{S}_{2}} C_{i j}}
$$

It is worth noting that above results can also be used in both decode-forward and multi-hop transmissions only with a different $C_{i j}$ in each protocol.

\section{Delay Analysis With Interference Subtraction}

In order to further investigate the interference impact on network performance, we consider a more realistic network scenario which allows multi-node transmissions along the same route using space time reuse scheme. To tackle the interference, the information of the sets of transmitters in each time slot is needed. In order to simplify the problem and get more meaningful results, here we use a linear network topology in which infinite nodes are regularly placed and each node on the route always has data to send. Therefore, given any transmission schedule, each node along the route will experience the same SINR. Specifically, the distance between adjacent nodes is normalized as 1 and the number of hops between the source and the destination is $H$.

We assume all nodes along the route transmit in the same frequency band and employ a regular TDM-schedule of length $K$-hops so that in time slot $\mathrm{t}$, the nodes $i K+(t \bmod K)$ are allowed to transmit, for $i=\ldots-1,0,1 \ldots$ It is still assumed that the data is transmitted from the source to the destination via multi-hop transmission without queuing delay.

\section{A. Interference Subtraction}

Consider in a four-hop linear network illustrated in Fig. 1, it is assumed that the source transmits a set of data packets one by one to the destination in multi-hop manner, we observe that in time slot T3, node $C$ forwards packet $m_{2}$, which is received by node $\mathrm{B}$ in $\mathrm{T} 2$, to node $D$. Node $B$ can actually keep a copy of the transmitted message $m_{2}$ locally, thus it knows the message being transmitted by node $C$ in T3 and can apply MUD with the stored prior information $m_{2}$ to mitigate the interference caused by node $C$, while node $\mathrm{A}$ is allowed to transmit another packet $m_{3}$ at the same time.
According to the system model assumed at the beginning of this section, the received SINR at each node is derived as

$$
\mathrm{SINR}=\frac{w}{N_{0}+\sum_{i=1}^{\infty}(i K+1)^{-\alpha} w+\sum_{i=1}^{\infty}(i K-1)^{-\alpha} w},
$$

where $K$ is the channel reuse factor and $w$ is the transmission power. After we implement the overlapped transmission, the received SINR can be improved as

$$
\mathrm{SINR}^{\prime}=\frac{w}{N_{0}+\sum_{i=1}^{\infty}(i K+1)^{-\alpha} w} .
$$

In the case of Fig. 1, we can derive that $\operatorname{SINR}^{\prime}(K=$ $2)=\operatorname{SINR}(K=4)$, which means that employing MUD [3] in wireless networks can help increase spatial reuse without losing system performance (e.g., delay). Motivated by the fact that prior information available at the receiver can be utilized to achieve perfect interference subtraction by using MUD scheme and therefore invite more simultaneous transmissions along a multi-hop routing, we propose here to further exploit delay performance in cooperative networks by employing MUD scheme.

Theorem 1: For a regular linear network scenario, the performance gain $g$, which is defined as the ratio of delay performance under multi-hop scheduling employing overlapped transmission and that without employing overlapped transmission, is bounded by

$$
\frac{A}{D}<g<\frac{B}{C}<1 .
$$

where $A=\ln \left(1+\frac{K^{\alpha}(K-1)^{\alpha}}{(1+\rho)\left(K^{\alpha}+(K-1)^{\alpha}\right) \operatorname{zeta}[\alpha]}\right), \quad B=$ $\ln \left(1+\frac{K^{\alpha}(K+1)^{\alpha}}{(1+\rho)\left(K^{\alpha}+(K+1)^{\alpha}\right) \operatorname{zeta}[\alpha]}\right), C=\ln \left(1+\frac{K^{\alpha}}{(1+\rho) \operatorname{zeta}[\alpha]}\right)$, $D=\ln \left(1+\frac{(K+1)^{\alpha}}{(1+\rho) \text { zeta }[\alpha]}\right), C \neq 0, D \neq 0, K$ is the channel reuse factor, $\alpha$ is the path loss exponent, $\operatorname{zeta}[2]=\frac{\pi^{2}}{6}$, zeta $[3]=1.202$ and zeta $[4]=\frac{\pi^{4}}{90}$.

Proof: See Appendix B.

In general, theorem 1 tells us the multi-hop scheduling employing overlapped transmission can achieve much better delay performance than that without employing overlapped transmission. For example, for the case where reuse factor $K=3$ and path loss exponent $\alpha=3$, the upper bound performance of gap ratio $g$ is 0.41 , which means up to $58.67 \%$ transmission time can be saved when using overlapped transmission.

\section{B. End-to-end Delay Analysis}

We assume that $L$ nats of data are transmitted in $m$ equal size packets through a multi-hop route using space time reuse scheme. Without considering the additional overheads in each packet, the end-to-end (ETE) delay in channel reuse is

$$
D_{E T E}=(H+(m-1) K) D_{p h},
$$

where $H$ is total number of hops between the source and the destination and $D_{p h}$ is the delay per hop. Here, by using the results in Section IV, the optimal end-to-end delay of different cooperative protocols with a constrained end-to-end reliability $P$ are the followings: 


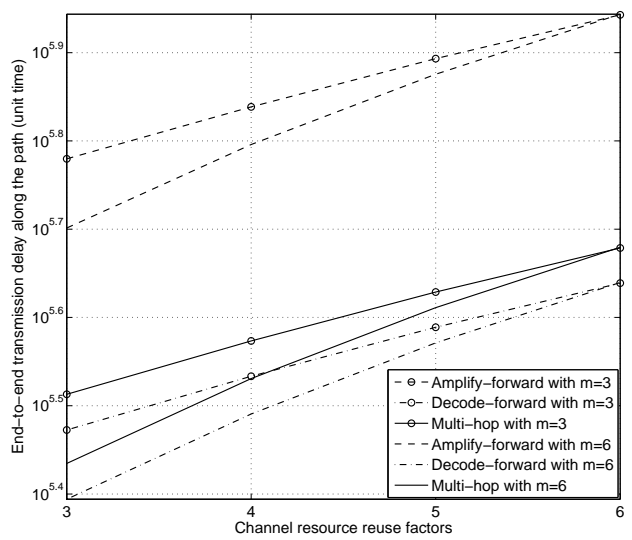

Fig. 2. End-to-end delay performance when using overlapped transmission

1) For amplify-forward: the end-to-end delay is derived as

$$
D_{E T E}^{A F} \geq \frac{(H+(m-1) K)\left(\rho \frac{L}{m}-\ln \left(\frac{2 P}{m H}\right)\right)}{\rho \ln \left(1+\frac{\operatorname{SINR}_{s, d}+f\left(\operatorname{SINR}_{s, r}, \operatorname{SINR}_{r, d}\right)}{1+\rho}\right)} .
$$

where $\operatorname{SINR}_{s, d}=\frac{w 2^{-\alpha}}{N_{0}+\sum_{i=1}^{\infty}(i K+2)^{-\alpha} w}$ and $\operatorname{SINR}_{s, r}=$ $\mathrm{SINR}_{r, d}=\frac{w}{N_{0}+\sum_{i=1}^{\infty}(i K+1)^{-\alpha} w}$.

2) For decode-forward: the end-to-end delay is

$$
D_{E T E}^{D F} \geq \begin{cases}\frac{(H+(m-1) K)\left(\rho \frac{L}{m}-\ln \left(\frac{2 P}{m H}\right)\right)}{\rho \ln \left(1+\frac{2 \operatorname{sIN}_{s, d}}{1+\rho}\right)}, & \text { if } R>I \\ \frac{(H+(m-1) K)\left(\frac{L}{m}-\ln \left(\frac{2 P}{m H}\right)\right)}{\rho \ln \left(1+\frac{\operatorname{sNR}_{s, d}+\operatorname{SINR}_{r, d}}{1+\rho},\right.}, & \text { if } R \leq I\end{cases}
$$

where $R=L /\left(2 m N_{p h} \ln 2\right)$ bit/s/unit hertz and the channel capacity $I=\frac{1}{2} \log \left(1+\operatorname{SINR}_{s, r}\right)$.

3) For multi-hop: the end-to-end delay is

$$
D_{E T E}^{M H} \geq \frac{(H+(m-1) K)\left(\rho \frac{L}{m}-\ln \left(\frac{P}{m H}\right)\right)}{\rho \ln \left(1+\frac{\operatorname{SINR}_{s, r}}{1+\rho}\right)} .
$$

where $\operatorname{SINR}_{s, r}=\frac{w}{N_{0}+\sum_{i=1}^{\infty}(i K+1)^{-\alpha} w}$. It is worth noting that the cooperative protocols are applicable when channel reuse factor $K>2$, since each cooperative transmission requires two receivers along the route. When $K \leq 2$, only multi-hop transmission is applicable.

The end-to-end delay performance of different cooperative protocols is shown in Fig. 2 as a function of channel reuse factor. It is assumed that total size of $4 \times 10^{4}$ nats of data are transmitted via a 6 hops route, the transmission power to noise ratio $\gamma=10 \mathrm{~dB}$, path loss exponent is set as $\alpha=3, \rho=0.9$ and the prefixed end-to-end reliability is $P=0.001$. It is of interesting to observe that choosing a larger block number $m$ leads to a better end-to-end delay performance. In other words, the original data divided in smaller block size is preferable to minimize delay. In addition, as the reuse factor increases, the whole transmission will experience a longer delay. There are two reasons that can explain this. First, according to (26), when $L$ is large, the numerator can be simplified as $\rho\left(K L\left(\frac{m-1}{m}\right)+\right.$ $\left.\frac{H L}{m}\right)$, in which $K$ will increase with a higher order than that

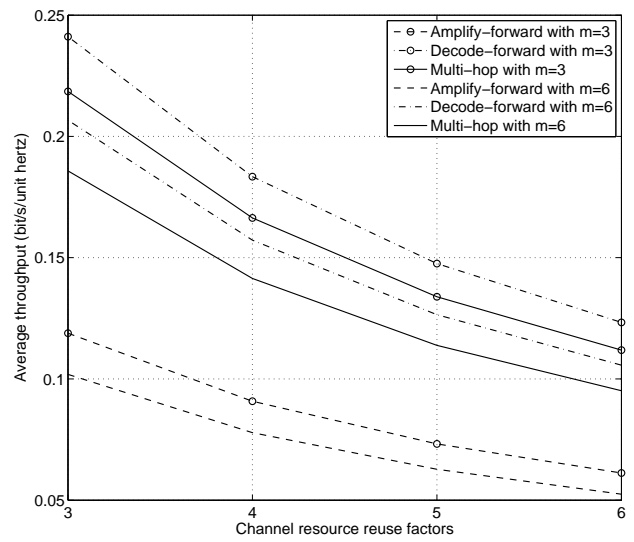

Fig. 3. Average throughput performance when using overlapped transmission

in the denominator. Second, since we are interested in low SNR cases, which means the interference will not domain the performance even when the reuse factor is small. Furthermore, when reuse factor approaches its maximum $(K=H)$, the endto-end delay will not be affected by the block number $m$ as it corresponds to the interference free scenario.

\section{Throughput Analysis}

In order to find more insights on the relation between the delay and any other system performance parameter (e.g., throughput), we are interested in addressing another relevant problem of maximizing the end-to-end throughput under the same network scenario. The average throughput can be expressed as

$$
R=\frac{L}{m K N_{p h}} .
$$

where $N_{p h}$ equals $D_{p h}$ is the coding length per hop. Under the same system setup, it is clear in Fig. 3 that the original data divided in larger block size can help achieve larger throughput. To gain some insights, we consider the optimal $m$ and $K$ in low SNR region, for example, using amplify-forward, it yields

$$
R \leq \underbrace{\frac{\frac{L}{m}}{\frac{L}{m}-\frac{1}{\rho} \ln \left(\frac{2 P}{m H}\right)}}_{\mathcal{S}_{1}} \underbrace{\frac{\ln \left(1+\frac{\operatorname{SINR}_{s, d}+f\left(\operatorname{SINR}_{s, r}, \operatorname{SINR}_{r, d}\right)}{1+\rho}\right)}{K}}_{\mathcal{S}_{2}} .
$$

The upper bound performance of throughput is divided into $\mathcal{S}_{1}$ and $\mathcal{S}_{2}$, respectively. In order to achieve the maximum value in (28), both $\mathcal{S}_{1}$ and $\mathcal{S}_{2}$ should be maximized. It is easy to verify that $\mathcal{S}_{1}$ is maximized when $m$ is as small as possible. In $\mathcal{S}_{2}$, since the numerator closes to 0 when SNR remains at a low level, the optimal $\mathrm{K}$ at the denominator should be the smallest as well.

A careful reader might notice that in fact there is a trade-off between the end-to-end delay and the network throughput. As block size of the original data decreases, the end-to-end delay is reduced correspondingly. However, the network throughput is adversely affected by small block size. Meanwhile, the 
channel reuse factor also plays an important role in system performance. Based on the power level that system selects, the optimal $K$ would be varied by other system parameters.

\section{CONCLUSION}

In this paper, we have investigated the transmission delay in terms of finite coding length derived from the random coding bound for different cooperative protocols. Moreover, by considering the interference impact on system performance, we have investigated performance gain on transmission delay for wireless cooperative networks by using overlapped transmission. Both analytical and numerical results are developed to show the significant improvement on the system performance by using cooperative transmission with overlapping as well as the trade-off between the end-to-end delay and network throughput.

\section{APPENDIX}

\section{A. Proof for (15) and (16)}

Define $x_{n}=P_{i j}^{D T}$ and $K_{n}=\frac{1}{\rho \ln \left(1+\frac{\operatorname{SINR}_{i j}^{n}}{1+\rho}\right)}$, where $n \in$ $\left[1, \cdots,\left|\mathcal{S}_{1}\right|\right]$. The first optimization problem in (14) can be written as

$$
\min \quad \sum_{n=1}^{\left|\mathcal{S}_{1}\right|} K_{n}\left(\rho L-\ln x_{n}\right), \quad \text { s.t. } \sum_{n=1}^{\left|\mathcal{S}_{1}\right|} x_{n} \leq z .
$$

According to the Kuhn-Tucker condition, the inequality constraints can be converted to the equality constrains, and the optimal solution of $x_{n}$ can be found from [8]

$$
-\frac{K_{\left|\mathcal{S}_{1}\right|}}{x_{\left|\mathcal{S}_{1}\right|}}+\lambda=0, \quad \lambda\left(\sum_{n=1}^{\left|\mathcal{S}_{1}\right|} x_{n}-z\right)=0
$$

Hence the Lagrange multiple and the optimal solutions of $x_{n}$ should be

$$
\lambda=\frac{1}{z} \sum_{n=1}^{\left|\mathcal{S}_{1}\right|} K_{n}, \quad x_{n}=\frac{z K_{n}}{\sum_{n=1}^{\left|\mathcal{S}_{1}\right|} K_{n}}
$$

Hence the total delay consumed by the links in the set, $\mathcal{S}_{1}$, will be

$$
\begin{aligned}
\sum_{i j \in \mathcal{S}_{1}} D_{i j}^{D T} & =\sum_{n=1}^{\left|\mathcal{S}_{1}\right|} K_{n}\left(\rho L-\ln x_{n}\right) \\
& =\sum_{i j \in \mathcal{S}_{1}} K_{i j}\left(\rho L-\ln \left(\frac{z K_{i j}}{\sum_{i j \in \mathcal{S}_{1}} K_{i j}}\right)\right)
\end{aligned}
$$

where the solution in (15) is obtained. Using the similar method, we can solve the second optimization problem.

\section{B. Proof for Theorem 1}

Consider the network scenario that multi-node transmissions are enabled along the same route using space time reuse scheme. The minimum delay per hop using non-overlapped transmission is

$D_{n o}=\frac{\rho L-\ln P_{p h}}{\rho \ln \left(1+\frac{w}{(1+\rho)\left(N_{0}+\sum_{i=1}^{\infty}(i K+1)^{-\alpha} w+\sum_{i=1}^{\infty}(i K-1)^{-\alpha} w\right)}\right)}$ and the minimum delay per hop using overlapped transmission is

$$
D_{o}=\frac{\rho L-\ln P_{p h}}{\rho \ln \left(1+\frac{w}{(1+\rho)\left(N_{0}+\sum_{i=1}^{\infty}(i K+1)^{-\alpha} w\right)}\right)},
$$

Assuming the system is in interference limited region, in which white noise power $N_{0} \ll w$, the delay performance gain is

$$
g=\frac{\mathcal{D}_{1}}{\mathcal{D}_{2}}=\frac{\ln \left(1+\frac{1}{(1+\rho)\left(\sum_{i=1}^{\infty}(i K+1)^{-\alpha}+\sum_{i=1}^{\infty}(i K-1)^{-\alpha}\right)}\right)}{\ln \left(1+\frac{1}{(1+\rho)\left(\sum_{i=1}^{\infty}(i K+1)^{-\alpha}\right)}\right)}
$$

1) $\mathcal{D}_{1}: \Rightarrow \ln \left(1+\frac{1}{(1+\rho)(\underbrace{\sum_{i=1}^{\infty}(i K+1)^{-\alpha}}_{\beta_{1}}+\underbrace{\left.\sum_{i=1}^{\infty}(i K-1)^{-\alpha}\right)}_{\beta_{2}})}\right.$

Then we have the following bounds

$$
(1+K)^{-\alpha} \sum_{i=1}^{\infty} i^{-\alpha}<\beta_{1}<K^{-\alpha} \sum_{i=1}^{\infty} i^{-\alpha}
$$

Since $\sum_{i=1}^{\infty} i^{-\alpha}$ is converged when $\alpha>1$, we can directly get the result from zeta function [9]. Therefore, $\beta_{1}$ is bounded by two finite boundaries.

We can derive the similar result for $\beta_{2}$, which is

$$
K^{-\alpha} \operatorname{zeta}[\alpha]<\beta_{2}<(K-1)^{-\alpha} \operatorname{zeta}[\alpha]
$$

2) $\mathcal{D}_{2}: \Rightarrow \ln \left(1+\frac{1}{\infty}\right)$

$$
(1+\rho)(\underbrace{\sum_{i=1}^{\infty}(i K+1)^{-\alpha}}_{\beta_{3}})
$$

We can derive the following

$$
0<(1+K)^{-\alpha} \operatorname{zeta}[\alpha]<\beta_{3}<K^{-\alpha} \operatorname{zeta}[\alpha]
$$

Hence the bound performance of $\mathcal{D}_{2}$ is

$\ln \left(1+\frac{K^{\alpha}}{(1+\rho) \operatorname{zeta}[\alpha]}\right)<\mathcal{D}_{2}<\ln \left(1+\frac{(K+1)^{\alpha}}{(1+\rho) \operatorname{zeta}[\alpha]}\right)$

Finally, the bound performance of $\frac{\mathcal{D}_{1}}{\mathcal{D}_{2}}$ can be directly derived from above.

\section{REFERENCES}

[1] J. N. Laneman, D. N. Tse, and G. W. Wornell, "Cooperative diversity in wireless networks: Efficient protocols and outage behavior," IEEE Trans. On Information Theory, vol. 50(12), Dec. 2004.

[2] I. E. Telatar and R. G. Gallager, "Combining queueing theory with information theory for multiaccess," IEEE Journal on selected Areas in Communication, vol. 13, pp. 963-969, August 1995.

[3] S. Boppana and J. M. Shea, "Overlapped transmission in wireless ad hoc networks," in International Conference on Communication, Circuits and Systems, 2006.

[4] J. Proakis, Digital Communication. McGraw-Hill, New York, 2000.

[5] R. G. Gallager, Information Theory and Reliable Communication. John Wiley and Sons, Inc.,, 1968.

[6] N. Wen and R. A. Berry, "Reliability constrained packet-sizing for linear multi-hop wireless networks," in IEEE International Symposium on Information Theory, July 2008.

[7] I. M. Gerhard Kramer and R. D. Yates, Cooperative Communications. Now Publishers Inc., Hanover, USA, 2006.

[8] S. Boyd and L. Vandenberghe, Convex optimization. Cambridge University Press, 2003.

[9] E. C. Titchmarsh and D. R. Heath-Brown, The Theory of the Riemann Zeta-function. Oxford University Press, USA, 1987. 